fy the indications for use, surgical application, advantages, pitfalls, limitations and complications of different implants, often with the collaboration of contributors closely involved in their design. Many chapters contain useful hints for those surgeons using the implants and are clearly written, with useful diagrams by authors who have experienced both the highs and lows associated with their use in spinal surgery.

However, the attempt to provide such a gazetteer leads to the inevitable flaw in such a text. In the rapidly evolving world of spinal implantation, no textbook will ever be able to provide a complete guide. This is exemplified by the book's omission of new developments in pedicle screw systems such as Pangea (Synthes SpineRRR) and Expedium (DePuy SpineRRR) and the complete omission of groups of commonly used implants; flexible stabilisation systems, such as the Dynesys (Zimmer SpineRRR) and Wallis (AbbottRRR) systems; and interspinous spacer devices such as the X-Stop (St Francis Medical TechnologiesRRR).

This text inevitably covers many implants that will gradually become superseded by technological and biomechanical advances. In the attempt to be comprehensive, the reader becomes somewhat 'lost'; for example, in the 20 chapters of different anterior cervical plates described in detail over 126 pages.

This is not to underestimate the text in the areas that it addresses well. The anatomical approach chapters are generally excellent - clear, concise and relevant to surgeons, not just anatomists. Particularly good chapters involve the anterior approaches to the cervical, lumbar and lumbosacral regions as well as the craniocervical junction. However, these could be accommodated in a much smaller text.

For a trainee preparing for a supervised operation using a new implant or an established consultant surgeon wishing to explore the range of implants on offer, this text offers some valuable guidance. Nevertheless, in a field moving so fast, one does wonder whether the authors' efforts would have been better placed in a more adaptable web-based resource.

A further point for us all to remember is that the implant used is only as good as the indication for which it is chosen and the skill and experience of the surgeon who applies it. Many surgeons frequently use implants for which they have derived significant training and have experience of their use in training programmes and specialist fellowships. In addition, this text should not be regarded as a shopping list for implants nor a training manual.

In summary, the text is a useful addition to the reference library of any spinal unit, especially in larger departments where there may be a range of implants frequently used. However, in view of the speed of developments, the authors are likely to find the need for addition and revision may come sooner than they wish.

\section{Cancer in the Spine: Comprehensive Care}

ROBERT MCLAIN, MAURIE MARKMAN, KAI-UWE LEWANDROWSKI, RONALD BUKOWSKI, ROGER MACKLIS, EDWARD BENZEL

$\begin{array}{ll}\text { EXTENT P/H } & 400 \mathrm{p} \text {, hardback } \\ \text { PRICE/ISBN } & £ 115.00 \quad 1588290743 \\ \text { PUBLISHER } & \text { Humana (New Jersey), } 2005 \\ \text { REVIEWER } & \text { Paul Thorpe } \\ \text { STAR RATING } & * * * *\end{array}$

The presentation of spinal tumours, especially metastases, to surgeons has increased significantly over the past few years. Recent research discussed mainly in oncology circles appears to have confirmed the important role of spinal surgery in palliative care, an area that was often dismissed as inappropriate in the past. Improvements in spinal reconstruction techniques, implants and peri-operative care have revolutionised the surgical options for patients with both primary and secondary spinal tumours.

This text is, therefore, well timed in its publication. The editor has assembled a broad range of North American authors representing most healthcare professions providing care for patients with spinal tumours, including oncologists, palliative care physicians, radiotherapists, surgeons, radiologists, pain specialists and physiotherapists. This gives the book a pleasing multidisciplinary outlook, reflecting the clinical co-operation that is essential to provide proper care for such patients.

The chapters are succinct and generally well referenced, giving this book appeal to a very broad range from the junior doctor embarking on an oncology or spinal surgical career to established clinicians seeking to refresh thinking on basic pathology or areas of care outside their immediate subspecialty. The basic science chapters are particularly well written - easy to cover but giving a broad understanding of, for example, the cell biology and growth of tumours. There are also useful chapters on the pathology and management of the most frequent pathological types of spinal tumour, including haematological malignancies.

The surgical techniques for spinal reconstruction in tumour surgery are complex and often challenging. The book touches on these, using authors well recognised as leaders in this field but does not claim to be comprehensive in its descriptions; it is not an operative manual. However, a slightly more in-depth analysis of surgical techniques, including en-bloc spondylectomy, would have made the text more attractive to the established spinal surgeon. The section on vertebroplasty and kyphoplasty usefully reminds the spinal surgeon that injection of cement into vertebra was originally developed for oncological applications rather than osteoporotic fractures, where it is most frequently used. It can be an excellent method of pain relief in spinal tumours, with the benefits of a minimally invasive approach. 
The nature of a text that tries hard to give a brief overview of all the different clinical facets of cancer care means that some themes will be underdeveloped. The chapter on investigating metastasis from an unknown primary appears overly negative in the ability to help such patients surgically and, therefore, shies away from full investigation on value-for-cost basis. As the availability of radiological investigations improves and their costs concurrently decrease, clinicians are finding increasing success in coming to a working diagnosis, thereby helping the planning of palliative treatment including surgery. It would also have improved the text to see more focus and probably a complete chapter on the outcome of different combinations of intervention, which is an area of particular current research interest.

Altogether, this text is a useful, well-written, referenced and edited overview of the basic tenets of spinal oncology practice. Its size and layout make it very easy to read and it can be recommended to any health professional forming part of an oncology team. It will also help to keep the improvements in spinal surgery and its outcome in the mind of all clinicians caring for patients with cancer. This, hopefully, will help to ensure that patients are not excluded from full assessment and potential treatments that are now thought significantly to improve the quality of limited life.

\section{Leg Ulcers: Diagnosis and Management (3rd edin)}

DAVID NEGUS, PHILIP COLERIDGE SMITH, JOHN BERGAN

\begin{tabular}{ll}
\hline EXTENT P/H & $354 \mathrm{p}$, hardback \\
PRICE/ISBN & $£ 99.00 \quad$ 0340810130 \\
PUBLISHER & Hodder (London), 2005 \\
REVIEWER & Madu Onwudike \\
STAR RATING & $* * *$
\end{tabular}

The title, Leg Ulcers: Diagnosis and Management, gives the impression that this text book has been restricted to the subject of leg ulcers. However, one soon discovers that it is a comprehensive text book of lower limb venous diseases.

Leg ulcers, particularly those of venous aetiology, constitute a major health problem in most Western countries. A significant proportion of the venous leg ulcer patients are relatively young, resulting in economic losses not only from the cost of treatment but also from the wastage of productive man-hours. As highlighted in the text book, the principal health personnel who manage venous leg ulcers vary between countries. In the UK, for example, the majority are initially seen and managed by nurses in community leg ulcer centres. This, notwithstanding the high profile given to venous diseases in general and leg ulcers in particular by the editors, is highly commendable since for most vascular units venous disease would contribute to at least $30 \%$ of their workload.

A detailed examination of normal anatomy and physiology of lower limb veins and a thorough discussion of pathology, pathological anatomy, pathophysiology, epidemiology and clinical presentation of all aspects of lower limb venous diseases have been systematically reviewed. Venous leg ulcers have been didactically presented with the specialist in mind. Extensive discussion of conventional and the more controversial views on pathophysiology have been appropriately discussed in a balanced format without the authors' personal bias influencing the quality of materials presented.

The basis for the accepted methods of investigation and treatment of venous leg ulcers, including compression, therapy and surgery are well presented. Unproven methods of treatment like pharmacotherapy, topical treatment and more modern methods including foam sclerotherapy, echosclerotherapy and endovenous laser treatment have also been adequately covered. There is also a very good chapter on 'The Differential Diagnosis of Leg Ulcers'. The text is illustrated using excellent line diagrams, black-andwhite as well as colour photographs. Each chapter has been very well researched with a comprehensive list of references.

Perhaps my only criticisms of the text book are the several instances of repetition and a few typographical errors. On the whole, however, it is a good specialist text book of lower limb venous diseases which any vascular surgeon with a special interest in this field would find quite useful. 